\title{
Scanning conductance microscopy investigations on fixed human chromosomes
}

\author{
Casper Hyttel Clausen, Jacob Moresco Lange, Linda Boye Jensen, Pranjul \\ Jaykumar Shah, Maria Ioannou Dimaki, and Winnie Edith Svendsen \\ MIC-Department of Micro and Nanotechnology, Technical University of Denmark, \\ Lyngby, Denmark
}

BioTechniques 44:225-228 (February 2008)

doi $10.2144 / 000112676$

Scanning conductance microscopy investigations were carried out in air on human chromosomes fixed on pre-fabricated $\mathrm{SiO}_{2}$ surfaces with a backgate. The point of the investigation was to estimate the dielectric constant of fixed human chromosomes in order to use it for microfluidic device optimization. The phase shift caused by the electrostatic forces, together with geometrical measurements of the atomic force microscopy (AFM) cantilever and the chromosomes were used to estimate a value for the dielectric constant of different human chromosomes.

\section{INTRODUCTION}

In recent years, the interest in micro total analysis systems ( $\mu$-TAS) for the sorting and characterization of biological samples has increased. Considerable efforts have been put into realizing such a device, and several simple systems have already been produced (1). The devices of the current generation of $\mu$-TAS rely on everything from cleverly designed microfluidic flow to light or electrical fields for manipulation and detection of the samples (2). Systems that currently use electrical readout are not optimal because the physical properties (e.g., size, permittivity, conductivity) of biological samples are mostly unknown. In order to optimize these devices, the physical properties need to be measured. This limited knowledge may be due to the biological samples' rather complex structure compared with fabricated solid state samples (nanowires/nanotubes, etc.). In this context, chromosomes are interesting in that they contain a very complex architecture of DNA and proteins, and chromosomal abnormalities may result in diseases such as Turner Syndrome and leukemia among others $(3,4)$. The physical properties of biological samples are important because they may be used in the design of micro systems that depend on electrotaxis and subsequent analysis of biological samples. One method for the study of physical properties is scanning probe microscopy (SPM), which has proven to be a flexible method for studying different sample types, both biological as well as solid state. Several SPM methods have already been used on chromosomes. Atomic force microscopy (AFM) (topography) imaging of stained $\mathrm{G}$ band patterns of the chromosomes was used to investigate the possibility of obtaining better resolution of the bands (5). Other reports of SPM applications on chromosomes include nearfield scanning optical microscopy measurements of the bands (6) and elasticity investigation of chromosome structure (7). One SPM method, scanning conductance microscopy (SCM), has in recent years been used to map out different properties of nano-sized particles, for example, the dielectric constant of nanoparticles of poly(ethylene oxide) (8), the conductance of carbon nanotubes and DNA (9), and trapped charges on a surface by carbon nanotubes (10). This makes SCM an interesting method for studying the electrical properties of small samples. As we have not been able to locate any reports of SCM used on chromosomes, we believe that this is the first time this method has been used on chromosomes.

\section{MATERIALS AND METHODS}

\section{Principle of the Measurements}

The principle and various applications of the SCM method have been well documented by M. Bockrath et al. (9), C. Staii et al. (8), and T.S. Jespersen et al. (10). The basic principle is that the tip of the AFM cantilever acts as a capacitor plate while a heavily doped Si layer with an insulating $\mathrm{SiO}_{2}$ layer on top acts as the other plate. The change in phase as the tip scans the samples deposited on top of the $\mathrm{SiO}_{2}$ is recorded. The data are used to estimate the dielectric constant of the sample. A sketch of the principle is shown in Figure 1.

The procedure of the SCM method used in this article is as follows: first a sample is dispersed on a $\mathrm{SiO}_{2}$ surface with a backgate, as shown in Figure 1. The SPM system performs a line scan along the surface of the sample in the dynamic mode using a conducting AFM tip. The tip is then raised several tens of nanometers and a new line scan is made following the topography trace of the previous scan. During the second scan a potential is applied between the tip and the doped layer. Because of the potential the phase in the second scan is different from the phase in the first. The only external force change on the AFM tip is the change in electric force. The phase difference depends on the cantilever parameters as well as the dielectric properties of the scanned objects, as was shown by $\mathrm{C}$. Staii et al. (8). It is further assumed that the tip of the AFM cantilever is a flat disk according to studies done by T.S. Jespersen et al. (10). The phase shift between the bare $\mathrm{SiO}_{2}$ surface and sample is mapped out and, according to C. Staii et al. (8) and T.S. Jespersen et al., (10) is governed by

$$
\Delta \phi \approx \frac{Q}{2 k}\left(\frac{\partial^{2} C_{1}}{\partial^{2} z}-\frac{\partial^{2} C_{2}}{\partial^{2} z}\right) V^{2},
$$


where $\Delta \mathrm{f}$ is the difference in the measured phase of the bare substrate and the fixed chromosomes, $C_{l}$ is the capacitance between the tip and backgate without a sample lying on the $\mathrm{SiO}_{2}, C_{2}$ is the capacitance between the tip and backgate with a sample lying on the $\mathrm{SiO}_{2}, z$ is the distance between the tip and sample (lift-height), $V$ is the potential difference between the tip and conducting layer, $Q$ is the quality factor of the AFM cantilever, and $k$ its spring constant. The second derivatives of the capacitances are given as

$$
\frac{\partial^{2} C_{1}}{\partial^{2} z}=\frac{2 \times \pi \times r_{t i p}{ }^{2} \times \varepsilon_{0}}{\left(z+d / \varepsilon_{S i O_{2}}\right)^{3}}
$$

and

$$
\frac{\partial^{2} C_{2}}{\partial^{2} z}=\frac{2 \times \pi \times r_{t i p}{ }^{2} \times \varepsilon_{0}}{\left(z+d / \varepsilon_{S i O_{2}}+h / \varepsilon_{p}\right)^{3}},
$$

where $r_{\text {tip }}$ is the radius of the tip, $\varepsilon_{0}$ the vacuum permittivity, $d$ the thickness of the oxide layer, $h$ the height of the particle, $\varepsilon_{\mathrm{SiO}_{2}}$ the permittivity of the oxide layer, and $\varepsilon_{p}$ the permittivity of the particle. The value of the quality factor of the cantilever is estimated by a measurement of the resonance frequency. The tip radius is estimated by the scanning electron microscopy images, and the spring constant is estimated by measuring the deflection of the AFM cantilever caused by another cantilever with a well-defined spring constant.

The lift-height $z$ is estimated to be around a few tens of nanometers according to theoretical studies for optimal signal carried out by J. Colchero et al. (11). Further, J. Colchero et al. (11) showed that the phase shift due to beam and cone of the AFM cantilever is almost constant compared to the contribution from the tip.

\section{Experimental Methods}

Substrates were fabricated by the use of four-inch heavily p-doped silicon wafers. A $100 \mathrm{~nm}$ thick silicon oxide layer was grown on the substrates, the oxide on the back was removed by hydrofluoric acid (HF), and a $20 \mathrm{~nm}$ layer of titanium was evaporated on the backside followed by a $1000 \mathrm{~nm}$ layer of gold.

The suspension of chromosomes was prepared from human T-lymphocytes (Model no. ACC282; DSMZ, Braunschweig, Germany) by a variant of the method developed by Wray et al. (12). Briefly, the cells were grown in RPMI-1640 media (Model no. 61870010; Invitrogen, Taastrup, Denmark) supplemented with $10 \%$ fetal bovine serum (Model no. F9665; Sigma, Brondby, Denmark) at $37^{\circ} \mathrm{C}$ and a $5 \% \mathrm{CO}_{2}$ atmosphere. The cells were arrested in log-phase with 0.06 $\mu \mathrm{g} / \mathrm{mL}$ colcemide (Model no. D1925; Sigma) for $10 \mathrm{~h}$ and collected at $200 \times$ $g$ for $10 \mathrm{~min}$. After removing the media the cells were washed once with serumfree media and collected at $200 \times g$ for $10 \mathrm{~min}$. The media was removed and the pellet was exposed to $75 \mathrm{mM} \mathrm{KCl}$ (Model no. P9327; Sigma) at $37^{\circ} \mathrm{C}$, gently vortexed to resuspend the cells, and incubated at $37^{\circ} \mathrm{C}$ for $20 \mathrm{~min}$. The cells were collected at $200 \times g$ for 10 min and washed once with hexylene glycol buffer (1.0 M hexylene glycol [Model no. 112100; Sigma], $0.5 \mathrm{mM}$ $\mathrm{CaCl}_{2}$ [Model no. 21115; Sigma], and $0.1 \mathrm{mM}$ PIPES buffer at $\mathrm{pH}$ 6.7 [Model no. 80637; Sigma]) at $37^{\circ} \mathrm{C}$ before resuspending the cells in fresh hexylene glycol buffer. The final chromosome stock solution was obtained by forcing the suspension through a 23 $\mathrm{G}$ needle and purified by collecting unlysed cells at $200 \times g$ for $10 \mathrm{~min}$. To deposit chromosomes on a substrate, the chromosomes were collected at $7500 \times \mathrm{g}$ for $20 \mathrm{~min}$ and resuspended in fixative (3 volumes methanol [Model no. 179337; Sigma] + 1 volume acetic acid [Model no. 320099; Sigma]). The

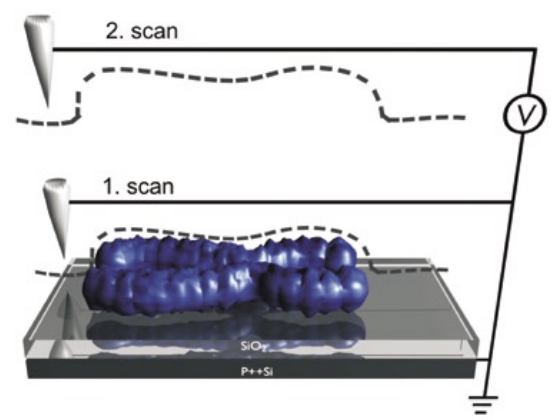

Figure 1. The principle of the SCM method. First a topography line scan is made, then the tip is lifted and another line scan is made with a potential difference between the AFM tip and the backgate.

chromosomes were finally deposited on the substrate by droplets and allowed to dry out at room temperature. The final resuspension in methanol/acetic acid was necessary in order to get sufficient and even spreading of the chromosomes. Using droplets of the hexylene glycol buffer resulted in a "pile" of chromosomes with salt residues on top because of the slow evaporation of hexylene glycol.

The scanning probe microscopy system used was a CP-II SPM system from Veeco (Breda, The Netherlands). AFM cantilevers were obtained from Budgetsensors (either model BSElectriMulti75 or BS-ElectriTap300; Nanoandmore, Wetzlar, Germany) (13), which both have a platinum coating. Analysis of the obtained data from the SPM system was carried out using the "SPIP" software (Image Metrology, Horsholm, Denmark) (14).

\section{RESULTS AND DISCUSSION}

The SPM images were obtained with a resolution of $512 \times 512$ pixels, with a scan rate of $0.6 \mathrm{~Hz}$, a potential difference of $2 \mathrm{~V}$, and lift-height of $30 \mathrm{~nm}$. The topography images were obtained by non-contact scans.

Topography and lift-mode phase images of different chromosomes with different cantilevers were obtained under ambient conditions. The phase shift and the corresponding chromosome height for each chromosome were obtained by taking the average across the middle of two of the arms of the chromosome (10 
A

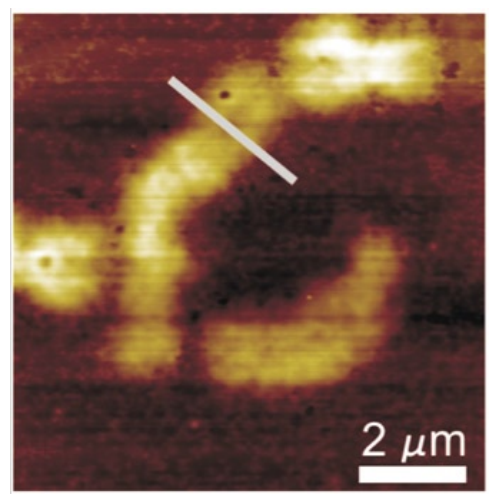

B

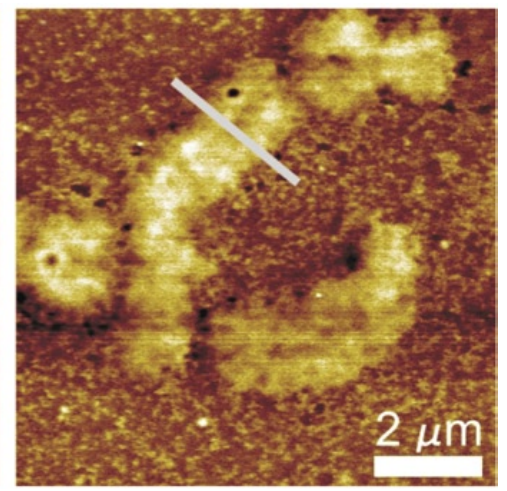

Figure 2. Topography and lift-mode phase images of different chromosomes. (A) topography image of chromosomes dispersed on a $\mathrm{SiO}_{2}$ surface with backgate; (B) phase shift image in lift-mode of the same chromosomes as in (A). The gray lines mark the line profiles across the chromosomes.

pixel to each side of the line profile [the gray line] in Figure 2, A and B). Using Equations 1, 2, and 3 the dielectric constant was estimated for each of the chromosomes. The chromosomes were investigated with different cantilevers with relative uniform resonance frequencies $( \pm 15 \mathrm{kHz})$. Five of the chromosomes were mapped out twice with a cantilever with a relative higher (around three times higher or 265.1 $\mathrm{kHz})$ resonance frequency. This was done to check if the dielectric constant changed due to the frequency of the cantilever.

Figure 3 shows the different dielectric constant obtained for different chromosomes with respect to their height. The error bars are calculated on the background of measurement uncertainties of the spring constant, the quality factor, the tip radius, and the oxide thickness. These have been calculated to be $\pm 5 \%$ to $\pm 8 \%$. Uncertainty values found for the lift-height and the sample height were $\pm 1 \%$, and for the potential difference an uncertainty of $\pm 5 \%$ was chosen.

As Figure 3 shows, the value for the dielectric constant is $10( \pm 4)$. Moreover the dielectric constant does not seem to vary with the chromosomes' height or the cantilever. Neither does the dielectric constant seem to vary with cantilever frequency. The flat curve of the dielectric constant for different chromosome height indicates that no artifacts influence the measurements. The spread in the values of the dielectric constant can be contributed to different effects. First of all, a chromosome is not a uniform material, rather it is composed of a complex mixture of proteins and DNA, and the dielectric constant may depend on the DNA-protein ratio at the point of measurement. This could give rise to the variation in the dielectric constant. Another thing that will influence the result is that the tip is not exactly flat but rather a bit curved. But according to researchers C. Staii et al. (8) and T.S. Jespersen et al. (10), who have reported good agreement between this assumption (flat tip) and experimental observations when investigating nanoparticles, this does not seem to be the obvious case. Finally, the sample preparation may also affect the chromosomal integrity, which could influence the measured dielectric constant. For example, the chromosomes' suspension will contain other biological material; the layer is not uniform and the layer thickness of the material is small (around $5 \mathrm{~nm}$ ) compared with the chromosomes, making it hard to make a good prediction of the impact on the measured data.

\section{Conclusion}

We have used SCM to investigate electrical properties of chromosomes fixed on a surface. We have estimated a value of the dielectric constant of the fixed chromosomes to be around 10. This was done by estimating the

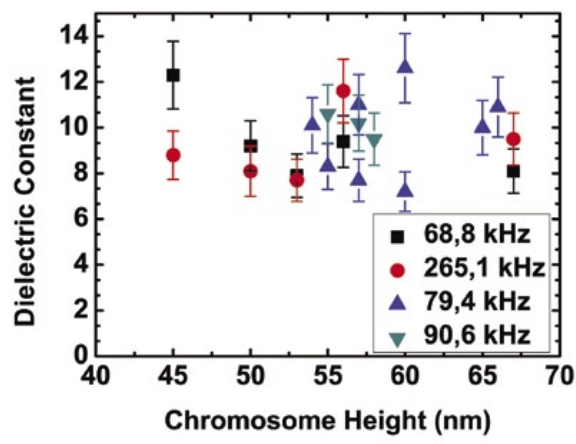

Figure 3. Measured dielectric constant of different chromosomes as a function of the height of the chromosome in question. properties of the AFM cantilever (quality factor, spring constant, and tip radius) and from the measured phase shift. We also used the assumption that the tip and surface act as a parallel plate capacitor. By these assumptions we have estimated a value of the dielectric constant of the fixed chromosomes, which was found to be around 10. Future studies will compare the influence of different preparation methods on the dielectric constant and include tests of this method in liquid. This work was guided by our interest in automated microsystems for chromosome analysis, which has led us to estimate the dielectric constant of chromosomes for later electric manipulation. It is believed that this method in the future can be used to characterize different biological samples and that the result will be used in design of future devices for biological sample detection.

\section{ACKNOWLEDGMENTS}

The authors would like to thank our colleagues for stimulating discussions. This work is supported by the Lundbeck Foundation.

\section{COMPETING INTERESTS STATEMENT}

The authors declare no competing interests. 


\section{REFERENCES}

1. Breslauer, D.N., P.J. Lee, and L.P. Lee. 2006. Microfluidics-based systems biology. Mol. Biosyst. 2:97-112.

2. Dittrich, P.S., K. Tachikawa, and A. Manz. 2006. Micro total analysis systems. latest advancements and trends. Anal. Chem. 78:3887-3907.

3. C. E. Ford, K. W. Jones, P. E. Polani, J. C. Dealmeida, and J. H. Briggs. 1959. A sexchromosome anomaly in a case of gonadal dysgenesis (Turner's syndrome). Lancet 1 : 711-713.

4. Nowell, P.C. and D.A. Hungerford. 1960. Minute chromosome in human Chronic granulocytic leukemia. Science 132:1497.

5. Tamayo, J. 2003. Structure of human chromosomes studied by atomic force microscopy Part II. Relationship between structure and cytogenetic bands. J. Struct. Biol. 141:189-197.

6. Moers, M.H.P., W.H.J. Kalle, A.G.T. Ruiter, J.C.A.G. Wiegant, A.K. Raap, J. Greve, B.G. De Grooth, and N.F. Van Hulst. 1996. Fluorescence in situ hybridization on human metaphase chromosomes detected by near-field scanning optical microscopy. J. Microsc. 182:40-45.
7. Nomura, K., O. Hoshi, D. Fukushi, T. Ushiki, H. Haga, and K. Kawabata. 2005. Visualization of elasticity distribution of single human chromosomes by scanning probe microscopy. Jpn. J. Appl. Phys. 44:54215424.

8. Staii, C., A.T. Johnson, Jr., and N.J. Pinto. 2004. Quantitative analysis of scanning conductance microscopy. Nano Lett. 4:859-862.

9. Bockrath, M., N. Markovic, A. Shepard, M. Tinkham, L. Gurevich, L.P. Kouwenhoven, M.W. Wu, and L.L. Sohn. 2002. Scanned conductance microscopy of carbon nanotubes and 1-DNA. Nano Lett. 2:187-190.

10. Jespersen, T.S. and J. Nygård. 2005. Charge trapping in carbon nanotube loops demonstrated by electrostatic force microscopy. Nano Lett. 5:1838-1841.

11. Colchero, J., A. Gil, and A.M. Baró. 2001. Resolution enhancement and improved data interpretation in electrostatic force microscopy. Phys. Rev. B 64:245403-245414.

12. Wray, W. and E. Stubblefield. 1970. A new method for the rapid isolation of chromosomes, mitotic apparatus, or nuclei from mammalian fibroblasts at near neutral $\mathrm{pH}$. Exp. Cell Res. 59:469-478.

13. www.nanoandmore.com

14. www.imagemet.com
Received 12 September 2007; accepted 16 October 2007.

Address correspondence to Casper Hyttel Clausen, MIC-Department of Micro and Nanotechnology, Technical University of Denmark, Building 345 East, DK-2800 Kgs. Lyngby, Denmark.e-mail: chc@mic. dtu.dk

To purchase reprints of this article, contact: Reprints@BioTechniques.com

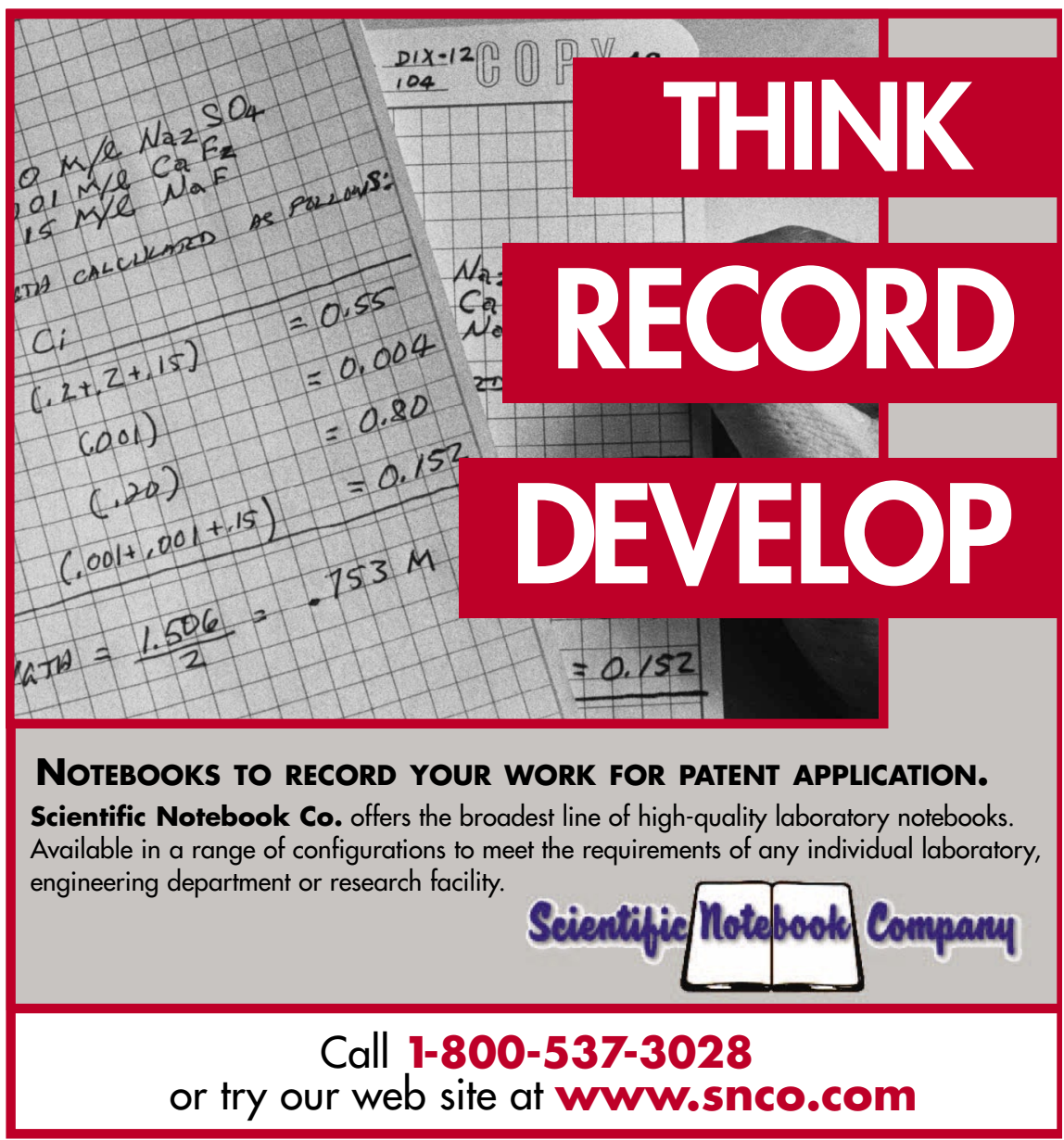

